\title{
Erratum to: A computational study of the wing-wing and wing-body interactions of a model insect
}

\author{
Xin Yu • Mao Sun
}

Published online: 11 November 2009

(C) The Chinese Society of Theoretical and Applied Mechanics and Springer-Verlag GmbH 2009

Erratum to: Acta Mech Sin (2009) 25(4):421-431

DOI 10.1007/s10409-009-0266-2

The corresponds address of the first author Xin Yu for the research paper "A computational study of the wing-wing and wing-body interactions of a model insect" published in Acta Mechanica Sinica (August 2009, Vol. 25, No. 4:421431) should be corrected as "Institute of Applied Physics and Computational Mathematics, 100191 Beijing, China”. The author apologizes for the negligence in checking the print proof.

The online version of the original article can be found under doi:10.1007/s10409-009-0266-2.

\section{$\mathrm{X} . \mathrm{Yu}$}

Institute of Applied Physics and Computational Mathematics,

100191 Beijing, China

M. Sun ( $₫)$

Institute of Fluid Mechanics, Beihang University,

100191 Beijing, China

e-mail: m.sun@263.net 